\title{
Kognitive Störungen bei älteren Patienten mit Niereninsuffizienz
}

\author{
Ute Hoffmann
}

\section{Was ist neu?}

- Prävalenz und Ursachen: Die Prävalenz von kognitiven Störungen bei Patienten mit Niereninsuffizienz ist hoch: Wurde genau getestet, ergaben sich Prävalenzzahlen von bis zu $87 \%$.

- Screening-Tests: Ein Screening kann mittels einfacher und schnell durchzuführender Tests erfolgen. Einige wurden zuletzt gerade bei Patienten mit Niereninsuffizienz als sehr sensitiv beschrieben.

- Differenzialdiagnostik: Kognitive Störungen mit behandelbaren nicht-vaskulären und nicht-neurodegenerativen Ursachen (Pseudodemenz) finden sich bei Patienten mit Niereninsuffizienz deutlich häufiger als bisher vermutet. Eine Identifizierung der Ursachen dieser demenziellen Syndrome ist deshalb äußerst wichtig, um eine kausale Therapie einleiten zu können.

- Therapiemöglichkeiten: Für Patienten mit chronischer Niereninsuffizienz gibt es gerade während der Hämodialysesitzungen zunehmende Möglichkeiten, ein kognitives Training durchzuführen. Alle Antidementiva zur Therapie der Alzheimer-Erkrankung mit Demenz sind bei Patienten mit Niereninsuffizienz inzwischen zugelassen und in der Regel gut verträglich.

- Dialyse bei Demenz: Die Einleitung einer chronischen Dialysetherapie muss bei demenzkranken Patienten kritisch evaluiert werden, da die Ziele, den funktionellen Status oder die Lebensqualität zu verbessern, in den neuen Studien meistens nicht erreicht wurden.

\section{Stand der Dinge}

Die Anzahl der älteren Menschen und parallel dazu die Anzahl der älteren Patienten mit chronischer Niereninsuffizienz nimmt aufgrund des demografischen Wandels stetig zu. Bei diesen älteren Patienten mit chronischer Niereninsuffizienz liegen im Vergleich zu der gleichaltrigen nierengesunden Population überproportional häufig kognitive Störungen vor. Diese beeinträchtigen nicht nur erheblich die Lebensqualität der Patienten, sondern gefährden auch deren Therapieadhärenz, z.B. wenn Tabletten nicht mehr korrekt eingenommen oder Ernährungs- und Trinkmengenempfehlungen nicht eingehalten werden. Desweiteren steigt durch kognitive Störungen das Risiko von funktionellen Einschränkungen sowie von stationären Aufenthalten deutlich [1,2].

\section{Prävalenz und Ursachen}

Die genaue Prävalenz von kognitiven Störungen bei Niereninsuffizienz lässt sich schwer abschätzen, da in den Studien, die zu diesem Thema verfügbar sind, unterschiedliche diagnostische Tests herangezogen wurden. Je niedriger die glomeruläre Filtrationsrate war und je genauer und ausführlicher gestestet wurde, desto höher war in den verschiedenen Studien die Prävalenz von kognitiven Störungen bei älteren Patienten. Für das Vorliegen einer leicht- bis schwergradigen kognitiven Einschränkung liegen uns deswegen variierende Prävalenzzahlen von $30-87 \%$ bei chronischer Niereninsuffizienz vor [3-5].

Es existieren viele Hypothesen, warum kognitive Einschränkungen bei Niereninsuffizienz-Patienten so häufig sind. Zum einen finden sich natürlich Erkrankungen wie die arterielle Hypertonie und der Diabetes als Risikofaktoren für vaskuläre Schäden, die sich zerebral als Mikroblutungen, Ischämien und Leukenzephalopathien manifestieren und zur sogenannten vaskulären Demenz führen können. Kürzlich konnte zudem gezeigt werden, dass eine Abnahme der glomerulären Filtrationsrate mit einer verminderten zerebralen Perfusion verbunden ist. Für die nichtklassischen Risikofaktoren des vaskulären Schadens aber auch der endothelialen Dysfunktion sind die chronische Inflammation, die Hyperkoagulabilität und der oxidative Stress bei chronischer Niereninsuffizienz zu nennen. Allerdings tritt bei Patienten mit Niereninsuffizienz nicht nur die vaskuläre Demenz, sondern auch die AlzheimerErkrankung mit Demenz deutlich häufiger auf, sodass auch neurodegenerative Hypothesen diskutiert werden. Verschiedene Urämietoxine wurden für neuronale Schädigungen bei Niereninsuffizienz verantwortlich gemacht $[3,6]$.

\section{Klinische Relevanz \\ Je schlechter die Nierenfunktion war und je genauer getestet wurde, desto höher lag in Studien die Prävalenz von kognitiven Störun- gen bei Patienten mit chronischer Niereninsuf- fizienz.}

\section{Screening-Tests}

Zur Einschätzung von kognitiven Störungen ist bei chronischer Niereninsuffizienz z.B. der MiniMental-Status-Test (MMST) einfach durchzuführen. Mit diesem Test können in ca. 10 Minuten kognitive Funktionen überprüft werden und bei einem Maximalwert von 30 Punkten eine Einteilung in leichte, mittelschwere und schwere Demenz erfolgen. Bei beginnender Demenz sind Tests wie der DemTect (Demenz-Detektion) oder bei Hämodialysepatienten der MoCA-Test (Montreal Cognitive Assessment) als sensitiver be- 
schrieben [7]. Auch wenn es praktisch wäre: Die Tests sollten möglichst nicht während Hämodialysesitzungen durchgeführt wurden, da bekannt ist, dass die Kognition im Verlauf der Hämodialysesitzung deutlich abnehmen kann [3, 5].

\section{Klinische Relevanz \\ Screening-Tests für Demenz sind bei Patienten mit chronischer Niereninsuffizienz einfach und schnell durchzuführen. Sie sollten allerdings außerhalb von Hämodialysesitzungen stattfinden.}

\section{Differenzialdiagnose der Demenz}

Der Sinn einer ausführlichen Diagnostik liegt in der Identifizierung von demenziellen Syndromen mit nicht-vaskulären und nicht-neurodegenerativen Ursachen, die kausal behandelbar sind. Eine kürzlich publizierte Studie, bei der bei $90 \%$ der Patienten eine Niereninsuffizienz vorlag, zeigte, dass potenziell behandelbaren Ursachen deutlich häufiger vorhanden sind als bisher vermutet. Bei $31 \%$ der Patienten mit Erstdiagnose Demenz und bei $18 \%$ der Patienten mit bereits bekannter Demenz lag z.B. die depressive Pseudodemenz neben vielen anderen als behandelbare Ursache vor. Gerade bei älteren Patienten mit Niereninsuffizienz ist aufgrund der Akkumulationsgefahr die Medikamentenanamnese äußert wichtig. Einige Medikamente können aufgrund einer zu starken Blutdrucksenkung zu kognitiven Einbußen führen [8]. Dazu zählen z. B.

- Antihistaminika, die häufig bei urämischem Pruritus eingenommen werden,

- Anticholinergika wie trizyklische Antidepressiva und Neuroleptika,

- sedierende Pharmaka wie z.B. Benzodiazepine,

- Glukokortikoide oder

- Antihypertensiva.

Bei einer Niereninsuffizienz ist es darüberhinaus essenziell, den Volumenstatus zu bestimmen, da ein zu niedriges Sollgewicht mit Exsikkose ebenfalls mit kognitiven Einschränkungen verbunden sein kann [3, 4, 6]. $>$ Tab. 1 zeigt einen Vorschlag für eine Differenzialdiagnostik.

\section{Klinische Relevanz}

Es sollte unbedingt die Identifizierung von demenziellen Syndromen mit nicht-vaskulären und nicht-neurodegenerativen Ursachen erfolgen, da diese bei Patienten mit Niereninsuffizienz häufig auftreten und ggf. kausal behandelbar sind.

\section{Therapie der Demenz}

Kognitives Training | Für Dialysepatienten gibt es viele Möglichkeiten eines kognitiven Trainings,

\author{
Untersuchungen \\ Labor: Natrium, Kalzium, CRP, GOT, \\ TSH, Vitamin $B_{12}$, Folsäure, TSH, \\ Kreatinin, Harnstoff \\ Screening auf Depression \\ Screening auf Alkoholabusus

\section{Blutdruckmessung \\ Überprüfung antihypertensive \\ Medikation} \\ Klinische Untersuchung, ggf.
Sonografie \\ Medikamentenanamnese, insbeson- \\ dere: \\ - Antihistaminika \\ - Anticholinergika wie trizyklische \\ Antidepressiva \\ - Neuroleptika \\ - Opiate \\ - Glukokortikosteroide \\ - Benzodiazepine \\ - Antihypertensiva
}

Zerebrale Bildgebung

Normaldruckhydrozephalus? Infarkte? (Mikro-) Blutungen? Tumoren? Arterosklerotische Leukenzephalopathie?

gerade weil hierfür dreimal pro Woche mehrere Stunden während der Dialysesitzungen zur Verfügung stehen. Praktisch sind hier E-Books, da sie mit nur einer Hand zu bedienen sind. Es gibt aber auch Bettvorrichtungen für Laptops, mit denen z.B. Gedächtnistrainingsübungen durchgeführt werden können. Gespräche mit den Taxifahrern, dem Dialysepersonal und den Mitpatienten sollten unbedingt gefördert werden, da die Einbindung in einen sozialen Kontext und die Kommunikation äußerst wichtig in der Demenzprophylaxe sind. Ebenfalls gilt es als erwiesen, dass körperliches Training an der Dialyse die Durchblutung verbesserte und damit die kognitiven Leistungen fördert (z.B. Bettfahrräder oder Gummitrainingsbänder) $[8,9]$.

Antidementiva | Zur Therapie der leichten bis mittelschweren Alzheimer-Demenz sind bei Niereninsuffizienz die Acetylcholinesterasehemmer zugelassen:

- Donezepil,

- Rivastigmin und

- Galantamin.

Sie führen zu einer Parallelverschiebung des Krankheitsverlaufs um etwa 6-12 Monate. Es ist keine Dosisreduktion notwendig, da der Q0-Wert - also der Anteil der bioverfügbaren Dosisfraktion, der nicht über die Nieren eliminiert wird bei allen drei Substanzen hoch ist (Donezepil
Tab. 1 Differenzialdiagnostik demenzielles Syndrom, die bei NiereninsuffizienzPatienten mit kognitiven Einschränkungen durchgeführt werden sollte. 


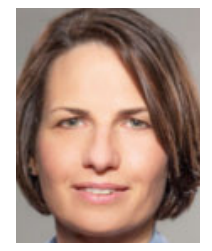

Prof. Dr. Ute Hoffmann ist Leitende Ärtzin der Abteilung für Nephrologie an der Klinik für Allgemeine Innere Medizin und Geriatrie im Krankenhaus Barmherzige Brüder Regensburg ute.hoffmann@ barmherzige-regensburg.de
Q00,95, Rivastigmin Q01,0, Galantamin Q00,8). Wichtig für die Wirksamkeit ist, dass im Verlauf die höchstmögliche Dosis angestrebt wird. Die Acetylcholinesterasehemmer sind bis auf selten auftretende gastrointestinale Nebenwirkungen sehr gut verträglich.

Bei mittelschwerer bis schwerer Alzheimer-Demenz bei chronischer Niereninsuffizienz kann in reduzierter Dosis der Modulator des N-Methyl-DAspartat (NMDA)-Rezeptors Memantin eingesetzt werden $(\mathrm{Q} 0$ 0,5). Memantin ist sehr gut verträglich, allerdings müssen die Patienten über die möglichen Nebenwirkungen Kopfschmerzen und Schwindel aufgeklärt werden $[10,11]$.

Dialyse I Das generelle Ziel der Nierenersatzverfahren, den Gesundheitszustand des Patienten zu verbessern oder aufrechtzuerhalten, wird bei älteren Patienten mit Demenz nur selten erreicht, vor allem wenn bereits eine fortgeschrittene funktionelle Abhängigkeit besteht $[12,13]$. In einer großen Studie von Dialysepatienten aus Pflegeheimen konnte ein Jahr nach Dialysebeginn der funktionelle Status nur bei $13 \%$ aufrecht erhalten werden, die Einjahresmortalität lag bei 58\% [1, 14]. Die Einleitung einer chronischen Dialysebehandlung bei Demenz muss deswegen mit allen Beteiligten gut überlegt werden.

Damit die Entscheidung, wann eine bereits begonnene chronische Dialysetherapie bei fortschreitender Demenz nicht weiter fortgeführt werden sollte, nicht in alleiniger Verantwortung der Angehörigen, der gesetzlichen Betreuer und der Ärzte bleibt, sollten Dialysepatienten zu Zeiten ihrer vollen mentalen Zurechnungsfähigkeit - am besten zeitgleich mit der Einleitung der Dialysetherapie - eine Patientenverfügung mit Vollmachtserteilung erstellen. Wenn der Patient diese nicht besitzt, sollte eine Lebensverlängerung durch die Fortführung der Dialyse immer dem mutmaßlichen Willen des Patienten entsprechen $[13,15]$.

\section{Klinische Relevanz}

Trotz Einleitung einer chronischen Dialysetherapie nimmt bei Patienten mit Demenz der funktionelle Status in der Regel rasch ab. Dialysepatienten sollten immer eine Patientenverfügung mit Vollmachtserteilung besitzen, in der klar hervorgeht, ob die Dialyse im Fall einer fortschreitenden Demenz weiter durchgeführt werden soll.

\section{Literatur}

1 Sarnak MJ, Tighiouart H, Scott TM et al. Frequency of and risk factors for poor cognitive performance in hemodialysis patients. Neurology 2013; 80: 471-480

2 Murray AM, Knopman DS. Cognitive impairment in CKD: no longer an occult burden. Am J Kidney Dis 2010; 56: 615-618

3 Bugnicourt JM, Godefroy O, Chillon JM et al. Cognitive disorders and dementia in CKD: the neglected kidney-brain axis. J Am Soc Nephrol 2013; 24: 353-363

4 Djukic M, Wedekind D, Franz A, Gremke M, Nau R. Frequency of dementia syndromes with a potentially treatable cause in geriatric in-patients: analysis of a 1-year interval. Eur Arch Psychiatry Clin Neurosci 2015; 265: 429-438

5 Yaffe K, Ackerson L, Kurella TM et al. Chronic kidney disease and cognitive function in older adults: findings from the chronic renal insufficiency cohort cognitive study. J Am Geriatr Soc 2010; 58 338-345

6 Sedaghat S, Vernooij MW, Loehrer E et al. Kidney Function and Cerebral Blood Flow: The Rotterdam Study. J Am Soc Nephrol 2015; pii: ASN.2014111118

7 Tiffin-Richards FE, Costa AS, Holschbach B et al. The Montreal Cognitive Assessment (MoCA) - a sensitive screening instrument for detecting cognitive impairment in chronic hemodialysis patients. PLoS One 2014; 9: e106700

8 Deuschl G, Maier W. S3-Leitlinie “Demenzen”. Langversion - 1. Revision, August 2015. Im Internet: https://www.dgppn.de/fileadmin/user_ upload/_medien/download/pdf/kurzversionleitlinien/REV_S3-leiltlinie-demenzen.pdf (Letzter Zugriff: 17.2.2016)

9 Heiwe $\mathrm{S}$, Jacobson $\mathrm{SH}$. Exercise training in adults with CKD: a systematic review and meta-analysis. Am J Kidney Dis 2014; 64: 383-393

10 Howard R, McShane R, Lindesay J et al. Donepezil and memantine for moderate-to-severe Alzheimer's disease. N Engl ] Med 2012; 366: 893-903

11 Haefeli WE. Dosing - Hilfsmittel zur ArzneimittelAnwendung \& -Sicherheit. Dosierung bei Niereninsuffizienz. www dosing de (Letzter Zugriff: 17.2.2016)

12 Ying I, Levitt Z, Jassal SV. Should an elderly patient with stage $V$ CKD and dementia be started on dialysis? Clin J Am Soc Nephrol 2014; 9: 971-977

13 Hoffmann U, Sieber C. Nierenersatztherapie im Alter - bei wem, wann, wie, wie lange? Dtsch Med Wochenschr 2014; 139: 1568-1571

14 Kurella TM, Covinsky KE, Chertow GM, Yaffe K, Landefeld CS, McCulloch CE. Functional status of elderly adults before and after initiation of dialysis. N Engl J Med 2009; 361: 1539-1547

15 Douglas C, Murtagh FE, Chambers El, Howse M, Ellershaw J. Symptom management for the adult patient dying with advanced chronic kidney disease: a review of the literature and development of evidence-based guidelines by a United Kingdom Expert Consensus Group. Palliat Med 2009; 23 : 103-110

\section{Interessenkonflikt}

Die Autorin gibt an, dass kein Interessenkonflikt besteht.

DOI 10.1055/s-0041-111113

Dtsch Med Wochenschr 2016; 141: 462-464

(c) Georg Thieme Verlag KG . Stuttgart · New York.

ISSN 0012-0472 